\title{
Corrigendum
}

\section{Evolutionary games and population dynamics: maintenance of cooperation in public goods games}

The analysis of our model for the maintenance of cooperation in public goods games based on a feedback between ecological dynamics and game dynamics was incomplete. In addition to the scenarios described in fig. 2 of our original text (Hauert et al. 2006), the following dynamic regimes are also possible if the interior fixed point $Q$ exists. For small multiplication factors $r$, an additional bifurcation occurs in which $Q$ changes from an unstable focus to an unstable node (figure 1a). The fact that the population is driven to extinction if $Q$ is unstable remains true. Similarly, for large $r$-values, the stable focus $Q$ turns into a stable node (figure $1 b$ ). As long as $Q$ is stable, cooperators and defectors can coexist, except for a small region (marked with an asterisk in figure $1 b$ ) where the population disappears because the system is unable to recover from low populations densities or high abundance of defectors. Finally, when the stable focus loses stability
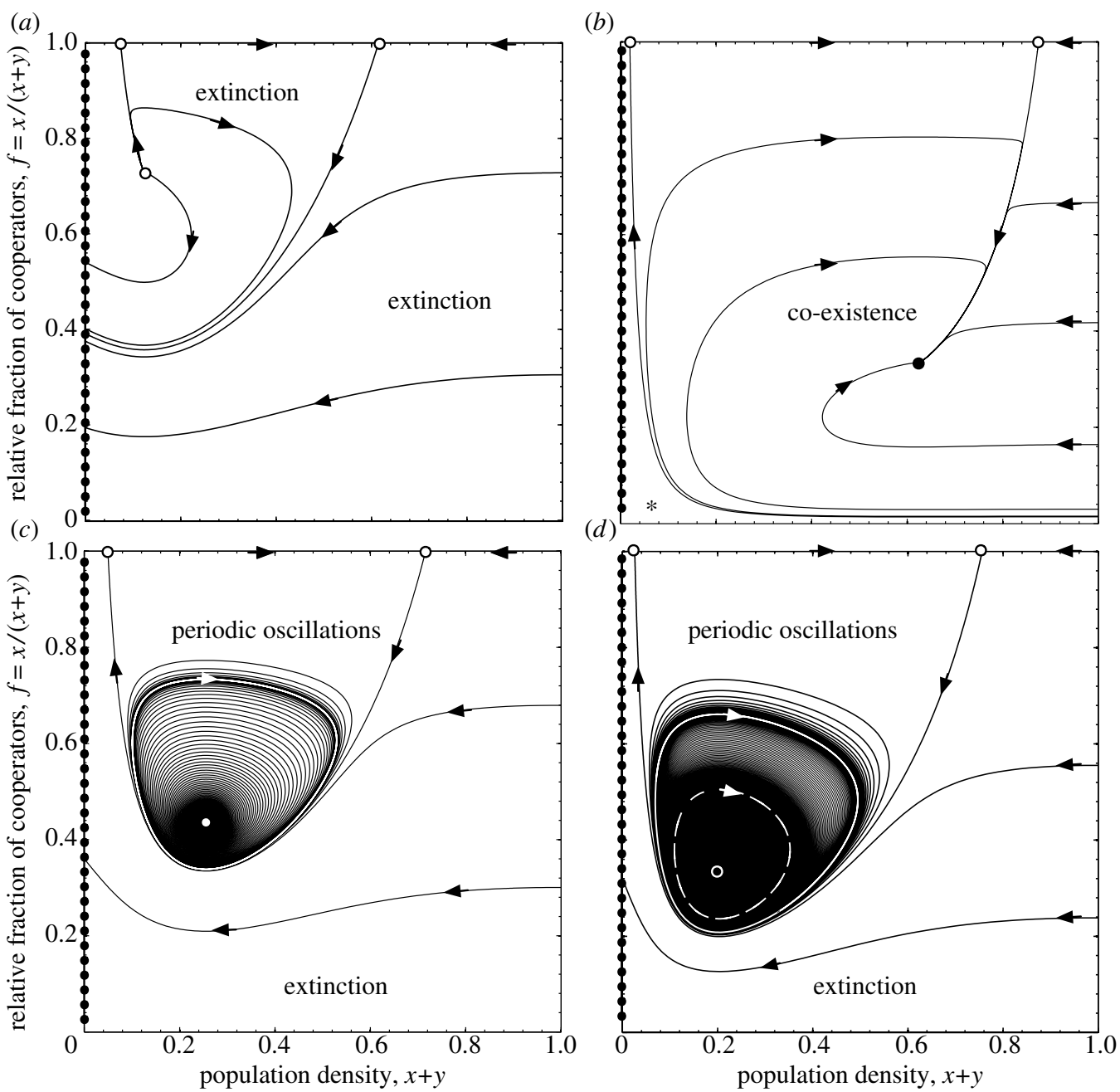

Figure 1. Additional dynamical scenarios for cooperation in public goods games with varying population densities. (a) For sufficiently small $r$-values, the interior fixed point $Q$ turns into an unstable node (open circle) before it disappears. (b) Conversely, for sufficiently high $r$-values, $Q$ turns into a stable node (filled circle). (c) The system exhibits stable limit cycles for a tiny region of $r$ just below the Hopf bifurcation that occurs at $r=N\left(N^{1 /(N-1)}-1\right)$. (d) More complex types of Hopf bifurcations are possible for larger $N$, including multiple stable (solid white line) and unstable (dashed white line) limit cycles. Parameters: $d=0.5, c=1 ;$ (a) $N=8, r=2.3 ;(b) N=8, r=5.0 ;(c) N=8, r=2.76$. $\left(r_{\mathrm{Hopf}} \approx 2.7672\right) ;(d) N=12, r=3.05\left(r_{\mathrm{Hopf}} \approx 3.0414\right)$. 
through a Hopf bifurcation, there is a very small range of $r$-values for which stable limit cycles occur and the system exhibits oscillatory coexistence of cooperators and defectors (figure 1c). As $r$ is decreased, the amplitude of the limit cycles increases quickly and the limit cycle disappears, giving rise to the extinction regime shown in fig. $2 c$ of our original text (Hauert et al. 2006). For larger group sizes $N$, more complicated types of Hopf bifurcations can be observed (e.g. leading to an unstable limit cycle separating a stable focus and a stable limit cycle, as shown in figure $1 d$ ), but the range of $r$-values where limit cycles occur remains very small. The oscillatory coexistence of cooperators and defectors is an interesting finding in itself, and the additional dynamic features reported here further support our original conclusion that ecological feedback can maintain cooperation in public goods games.

We thank J. Hofbauer and J. Wakano for comments.
Christoph Hauert $^{1, *}$, Miranda Holmes ${ }^{2, \dagger} \&$

Michael Doebeli ${ }^{2}$

${ }^{1}$ Program for Evolutionary Dynamics, Harvard University, One Brattle Square, Cambridge, MA 02138, USA

${ }^{2}$ Departments of Zoology and Mathematics, University of British Columbia, 6270 University Boulevard, Vancouver BC, V6T 1Z4, Canada

${ }^{\dagger}$ Present address: Courant Institute of Mathematical Sciences, New York University, 251 Mercer Street, New York, NY 10012, USA

*Author for correspondence (christoph_hauert@harvard.edu)

\section{REFERENCE}

Hauert, C., Holmes, M. \& Doebeli, M. 2006 Evolutionary games and population dynamics: maintenance of cooperation in public goods games. Proc. R. Soc. $B$ (doi:10.1098/rspb.2006.3600). 